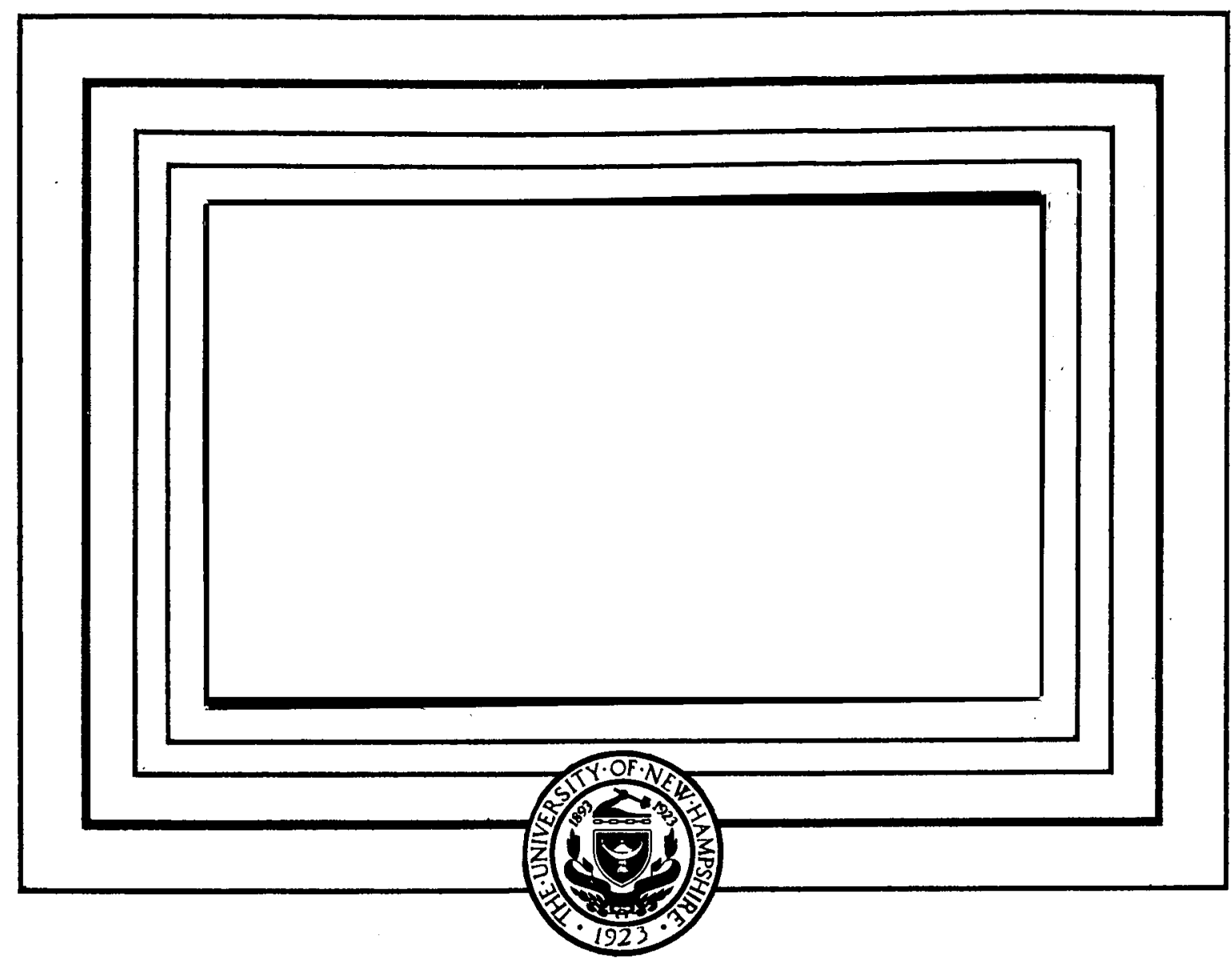

NASA - CR-122466 THE OSO-7 SPACECRAET (New Hampshire Univ.)

MUA RAY MONITOR FOR F. F. Higbie et al 5 Nov. $197124 \mathrm{p}$ CSCL $14 \mathrm{~B}$
N72-31457

Unclas G $3 / 14$

40500

\title{
tes
}

$462+22^{2} 22^{2}$

\section{Department of Physics}

UNIVERSITY OF NEW HAMPSHIRE

\author{
Durham
}




\section{A GAMMA RAY MONITOR FOR THE \\ OSO-7 SPACECRAFT* + \\ P. R. Higbie \\ E. L. Chupp \\ D. J. Forrest \\ I. U. Gleske}

November 5,1971

* This work was supported by NASA under Contract Nas 5-11054.

+ Presented at the IEEE Nuclear Science Symposium, November 3-5, San Francisco, California 
A GAMMA RAY MONITOR FOR THE OSO-7 SPACECRAFT

P. R. Higbie, E. I. Chupp, D. J. Forrest, I. U. Gleske

Department of Physics

University of New Hampshire

Durham, New Hampshire 03824

SUMMARY. A 3" x 3" NaI(T1) gamma ray ( 0.3 to $10 \mathrm{MeV})$ spectrometer with a CSI(Na) charged particle and anti-Compton shield has been developed for the ORBITING SOLAR OBSERVATORY (OSO-7) which was launched september 30, 1971. The instrument, designed for a rotating wheel compartment, utilizes a 377 channel quadratic PHA with accumulation times of 3,1 , or 0.5 minutes. Quick look and calibration data obtained via a direct data link to a minicomputer at the UNH campus allows near real time monitoring and control of the experiment. Various commands changing the operating mode can be executed. The functions which can be commanded include: rotation of the quadrants in which data is collected by $90^{\circ}$; gain adjustment of the central detector over a $6: 1$ range; manual or automatic sequencing of calibrations; variations of accumulation times by telemetering selected channels; and selection of reference directions. A small $x-r$ ay detector covering the range 7.5 to $120 \mathrm{keV}$ is also included.

\section{INTRODUCTION}

The primary objective for the University of New Hampshire instru- 
ment is monitoring the sun for emission of solar gamma rays. Possible gamma ray line emission from nuclear processes as well as the continuous gamma spectrum are to be investigated. The data can be correlated with sporadic solar events and can provide limits on steady or slowly varying gamma ray emissions. In addition, direct correlations can be made with data from the $x$-ray detector. These considerations led to an instrument which has high energy resolution. The instrument will also study the Earth's albedo gamma rays and such celestial sources as supernovae, their remnants, and the galactic disk.

Gamma ray lines will be emphasized in the spectrum because of the high resolution (7.5-8.0\% at $0.662 \mathrm{MeV})$. As an example, gamma rays at $0.51,2.22,4.43$, and $6.15 \mathrm{MeV}$ would indicate production of positrons, neutrons, and excited nuclei from various nuclear processes. The energy dependent angular resolution of the instrument will help in determining the contribution from the solar, albedo, and celestial sources.

A data link has been set up to provide near real time control for the instrument. The data can be scanned for calibrations and can be examined for evidence of solar activity. Indications of solar activity or predictions of high flare probabilities might prompt the use of the faster time resolution modes. 


\section{INSTRUMENT DESCRIPTION}

The instrument is shown schematically in Figure 1. The basic sensor is a 3" x 3" NaI(TI) crystal mounted on a RCA C31012 stacked ceramic ruggedized photomultiplier. This assembly is mounted inside a CsI (Na) anticoincidence shield viewed by RCA C70132B phototubes. An ancillary $\mathrm{X}$-ray detector and calibration sources complete the instrument. Figure 2 shows a photograph of the assembled flight instrument.

The NaI crystal was mounted by Harshaw Chemical Company directly on the sapphire face plate of the photomultiplier and hermetically. sealed in a thin-walled stainless steel housing (low radioactivity 304ss). The crystal was restrained within the housing by a spring delivering a force of $60 \pm 5$ lbs. Bleeder resistors were attached to the dynodes and coated with RTV 11. A rear housing was placed over the bleeder network and voids were foamed with Uralane 1723. The CsI(Na) cup was rigidly held against the outer aluminium housing by a pressure plate. A wave spring between the pressure plate and central detector restrained the latter from moving with respect to the cup.

The central detector is shielded by a thick CsI(Na) cup and a thin front slab of the same material. Charged particle interactions in either of these shields initiate anticoincidence 
logic signals. Gamma rays have a high probability of passing through a thin front shield without interaction; however, the cup being 1.5 inches thick nearest the central detector, attenuates $1 \mathrm{MeV}$ gamma rays by at least $60 \%$. Gamma rays which pass through the front slab and undergo compton scattering in the central detector have a high probability of interacting in the cup. A $0.511 \mathrm{MeV}$ escape gamma ray from an incident primary gamma ray producing a pair in the central detector may also interact in the cup. In either case an anticoincidence pulse is produced which improves the energy response function of the system by accentuating the photopeak. Figure 3 shows this effect for an external $\operatorname{co}^{60}$ source.

The CsI (Na) cup is viewed by four hemispherical RCA C70132B photomultipliers. Three tubes are arranged at approximately $120^{\circ}$ around the axis of symmetry, with the fourth tube at the apex of the cup. The cup is made from two cylindrically symmetric pieces with the parting plane near the fourth tube. The photomultipliers are coupled with RTV 165 to the shield crystals which are covered by household aluminium foil for enhancing light collection.

The front slab is viewed by a single RCA C70132B photomultiplier. One end of the slab is thickened and the hemispherical photocathode is recessed to be at the same level as the slab to take advantage 
of light piping effects. The $\mathrm{Am}^{241}$ doped NaI(T1) crystal winich forms part of the $\mathrm{X}$-ray calibration system is also viewed by this tube through the CsI, an air interface, and the glass hermetic seal of the $\mathrm{NaI}(\mathrm{T} I, \mathrm{Am})$ can.

The small X-ray monitor is an uncollimated NaI(Tl) cIYstal, 1/4" thick $\times 1$ 1/4" diameter, with a $10 \mathrm{mil}$ Be window. This is viewed by an RCA 4461 flat faced 1 1/2" diameter phototube. The output is divided into four energy channels: 7.5-15, $15-30,30-60,60-120 \mathrm{keV}$.

Calibration of the gamma ray spectrometer is accomplished by use of a $\mathrm{Co}^{60}$ source giving lines at 1.17 and $1.33 \mathrm{MeV}$. The source is dispersed (as cobalt octolate) in a plastic scintillator (Pilot M Partially Polymerized Plastic Scintillator) as described elsewhere (FORREST et al.). A $0.02 \mu \mathrm{Ci}$ source in the shape of a small disk was bonded to the end of a light pipe which passed through a hole in the CsI cup so that the source was positioned adjacent to the central detector. Light from the plastic scintillator is collected by an auxiliary C70132B phototube. When $\mathrm{Co}^{60}$. decays to $\mathrm{Ni} 60$, the beta particle is always accompanied by prompt gamma rays, more than $99 \%$ of which are the two cascade lines at 1.17 and $1.33 \mathrm{MeV}$. The gamma rays can pass unhindered into the central detector, but the beta particle deposits its

$$
-5-
$$


energy in the plastic scintillator thus giving a logic signal from the auxiliary phototube which is used to gate the signal from. the central detector. This system has achieved a beta ray tagging efficiency of better than 95\%. In normal operation the system is run in anticoincidence and the cobalt gamma rays are rejected, but in the calibration mode the system is run in coincidence and two prominent lines appear in the recorded spectrum with only accidental background contamination. A similar system to calibrate the $\mathrm{X}$-ray detector uses $\mathrm{Am}^{241}$ which emits an alpha particle in coincidence with a gamma ray line at $60 \mathrm{keV}$.

The instrument is located in compartment 9 of the rotating wheel section of osO-7 spacecraft with the crystal and cup axes colinear with a wheel radius. Data is accumulated when the instrument axis lies within $45^{\circ}$ of either the solar or antisolar direction. 


\section{INSTRUMENT DATA FORMATING}

A block diagram of our instrument is shown in Figure 4. The spacecraft telemetry system employs a $800 \mathrm{~Hz}$ clock and reads data out on a basic 32-word (byte) Main Frame cycle. The data from the instrument falls under three broad categories:

Spectral Data from the central detector which is read out in two words which appear in every Main Frame cycle. Flag, status, clock, and dead time words also accompany every spectrum transmitted.

X-ray Data is submultiplexed onto the Digital Subframe (DSF) which is a 48-word cycle on one word of the Main Frame. The instrument is assigned seven words which are spread through the cycle with two DSF cycles required to read out all $\mathrm{x}$-ray channels and associated flags and times. Every 7.7 seconds a half second "snapshot" of the sun is taken in the successive energy ranges. The comparison quadrant is treated similarly.

Housekeeping and Rate Data are telemetered in 10 consecutive words of the 48-word Analog Subcommutator Cycle (ASC).

\section{Gamma Spectral Data}

The gamma ray pulses from the anode of the central detector flow 
through the front end electronics, where they are amplified by a charge sensitive pre-amplifier. The output is double differentiated and passes through a linear gate controlled by a variety of logic pulses. The gate is strobed to pass the flat portion of the signal and a peak detector is used to charge a capacitor used by a Quadratic Analog to Digital Converter (QADC). The channel number $n$, which is the digitized output of this device is reiated to the energy loss, $\mathrm{E}$, in the central detector by

$$
E=k\left(n+n_{0}\right)^{2}
$$

Over the nominal energy range covered by the instrument the half width in energy of a crystal photomultiplier combination for a given gamma ray line is approximately proportional to $\sqrt{E}$. In terms of channel numbers, however

$$
\Delta E=2 k\left(n+n_{0}\right) \Delta n
$$

to first order in $\Delta \mathrm{n}$. Consequently, if we chose $\Delta \mathrm{E}$ to be half width, the corresponding $\Delta \mathrm{n}$ is approximately constant. Our system parameters were chosen such that we have approximately five channels under a gamma peak independent of energy. The QADC optimizes our telemetry usage by keeping the ratio of the detector to electronic resolution constant over the desired energy range (0.3-9.1 MeV) which then requires 377 channels. The QADC and memory is described in a companion paper in this volume. (BURTIS et al.) 
For a given pulse height, E, a corresponding 12-bit word is fetched from core storage, incremented and replaced. This memory is operated according to an exchange buffer technique. While one half is being updated by the random gamma input, the second half is sequentially read out into the spacecraft telemetry system. These halves are again divided into sections for the solar and antisolar directions.

\section{Gamma Logic}

The admission of the desired gamma ray into the data stream is controlled by a linear gate in the following manner: First, a window discriminator, set to match the lowest and highest channels of the following QADC, provides strobing input to the gate. For the nominal gain setting of the central detector this provides thresholds of 0.3 and $9.1 \mathrm{MeV}$. Other logic signals include PHA busy, anticoincidence pulses from the cup, slab and the co60 source. The $\mathrm{Co}^{60}$ pulse as noted above, can be placed in coincidence for calibration operation. The anticoincidence pulse from the CsI cup has a variable width to cover the slow decay component of $\mathrm{CsI}(\mathrm{Na})$ after giant pulses. An analog dead time circuit sums all contributions to the dead time and increments its corresponding memory word after every 1.515 millisecond dead time accumulation. The dead time circuit also returns a pulse to cover its own operation. A final control pulse is provided 
by the quadrant generator. This is an electronic subsystem which provides control pulses to the linear gate, the memory, the X-ray logic and the rate meters whenever the detector axis lies within the $90^{\circ}$ sectors centered on either the solar or background directions. Data is accumulated for complete quadrants only and the total clock time for which accumulation was permitted is also sorted in core memory.

\section{Fast Time Resolution and Other Modes}

Three formats for channel readouts are available: a full scan mode; a short scan mode covering channels selected for posssible lines at $0.511,2.2 \mathrm{MeV}$ and calibration lines; and an intermediate scan covering these lines and the 4.43 and $6.13 \mathrm{MeV}$ lines. The motive is to provide faster time resolutions of 30 and 61 seconds compared with the three minutes required for the full scan.

The instrument can be commanded to operate under several conditions besides the time read out and calibration modes described above. The night reference direction which is normally given by a Sun-pointing gyro can be switched to the magnetometer signal provided by the spacecraft. The quadrant normally aligned along a reference direction may be rotated by $90^{\circ}$. The central detector's gain can be controlled by adjusting its high voltage. Adjustments can be made in two ranges of approximately 150 volts 
Which slightly overlap. Each range is divided into 64 steps. This supply and the high voltage supply for the shields; Co 60 calibration, and $\mathrm{X}-\mathrm{r}$ ay tubes may be turned on and off independently. Calibration is automatically sequenced (but may be inhibited by command) at satellite dawn and dusk crossings and may also be initiated by a manual calibrate command. In the manual calibrate case, electronic pulses are also sequenced to calibrate the system amplifiers and thresholds.

\section{$\underline{x-r a y ~ S p e c t r a l ~ D a t a ~}$}

The pulses from the $x$-ray tube are amplified by a charge sensitive pre-amplifier and place either in coincidence or anticoincidence with the front slab, depending on whether the instrument is in the calibrate mode or not. The $\mathrm{x}-\mathrm{ray}$ data is also gated by the quadrant generator to accumulate only the latest complete quadrant. The analog pulses are attenuated by successive factors of two depending on which of the four energy intervals is being sampled and presented to a window discriminator. The number of counts is accumulated by an adaptive scalar which can handle a maximum of 18,688 pulses.

Rates and Housekeeping

Various rates and housekeeping data are handled via the analog subcommutator channel. These include log rate meter outputs 
for the shield, cup and central detector, each of which is sampled synchronously with the spacecraft rotation and read out every 15.36 seconds. Temperatures, voltages, and status of the operating modes are also monitored and read out every 184 seconds. A typical calibration curve for the rate meters is shown in Figure 5. 
IV. DETECTOR PROFERTIES

The system was calibrated using $\mathrm{Na}^{22}, \mathrm{Cs}^{137}, \mathrm{Co}^{60}, \mathrm{Th}^{228}$ sources and a special $\mathrm{Cm}^{244}-\mathrm{Cl}^{13}$ source. The last source gives a monoenergetic line at $6.130 \mathrm{MeV}$ through the $\mathrm{C}^{13}(\alpha, n) 0^{16}$ * reaction. The peak channel for the gamma ray lines from these sources is shown as $\sqrt{E}$ vs. $n$ in Figure 6 for a typical gain setting.

The crystal and photomultiplier combination were carefully chosen to give a nominal energy resolution of 7.58 at $0.662 \mathrm{MeV}$. Because of the saturation effects in the central photomultiplier, the flight unit was provided with a nonlinear voltage divider network to assure signal linearity over our range of interest.

The number of channels corresponding to the half widths for the sources listed above is also shown in Figure 6. The salient characteristics of the isntrument are summerized in Table II. 


\section{ACKNOWLEDGEMENTS}

Antal A. Sarkady of the UNH Electrical Engineering Department contributed significantly to the design and testing during the breadboard and prototype development. The instrument was designed, fabricated, and tested in collaboration with Time-zero corporation under the successive supervision of the Project Managers, Robert Kobayashi and Donald Rose and the design team of J. Chapsky, R. Evelyn-Veere, and D. Aalami. Considerable support and assistance was given by the oso Project office under Project Director, John Thole and the Experiment Manager, John Donley.

\section{REFERENCES}

Burtis, D. W., D. Aalami, R. H. Evelyn-Veere, and A. A. Sarkady, "A Quadratic Pulse Height Analyzer for Space Applications", Presented at the IEEE Nuclear Science Symposium, November 3-5, San Francisco, to be published in the Proceedings of the meeting.

Forrest, D. J., P. R. Higbie, L. E. Orwig, and E. L. Chupp, "An Electronically Gated Gamma and X-Ray Calibration scheme", to be published. 
TABLE I

SUMMARY OF MODES

MODE

FUNCTION

QUADRANT

SCAN

MANUAL CALIBRATE

AUTOCALIBRATE

SHIELD HIGH VOLTAGES

CENTRAL HIGH VOLTAGES

GAIN CHANGE

GAIN STEP

ASPECT REFERENCE
NORMAL OR ROTATED $90^{\circ}$

SELECTION OF CHANNELS READ OUT

(FULL， INTERMEDIATE OR SHORT).

ELECTRONIC AND RADIOACTIVE CALIBRATIONS.

RADIOACTIVE CALIBRATION AT DAWN OR DUSK,

(ON/OFF).

(ON/OFF).

(UPPER/LOWER CENTRAL DETECTOR HIGH VOLTAGE RANGE).

(0 THRU 63 STEPS ON EITHER CENTRAL DETECTOR HV RANGE). MAGNETOMETER OR GYROSCOPE, 


\section{TABLE II}

SPECTRAL RANGE

SPECTRAL RESOLUTION

ANGULAR RESOLUTION

TIME RESOLUTION

EFFECTIVE AREA

BITS PER SECOND

POWER (NOMINAL)

WEIGHT

DETECTOR TYPE
0.3-9. I MEV (NOMINAL),

$8 \%$ O $0.662 \mathrm{KEV}: 377$ CHANNEL QUADRATIC PULSE HEIGHT ANALYZER.

$120^{\circ} \times 70^{\circ}$ FWHM AT L MEV IN SOLAR AND ANTISOLAR DIRECTIONS.

3 MINUTES, 51 OR 30 SECONDS,

$45 \mathrm{cM}^{2}$ (POINT SOURCE ON AXIS).

50.

3.5 WATTS,

74 LBS.

$3^{\prime \prime} \times 3 "$ NAI (TL) CRYSTAL IN CSI (NA) SHIELD, 


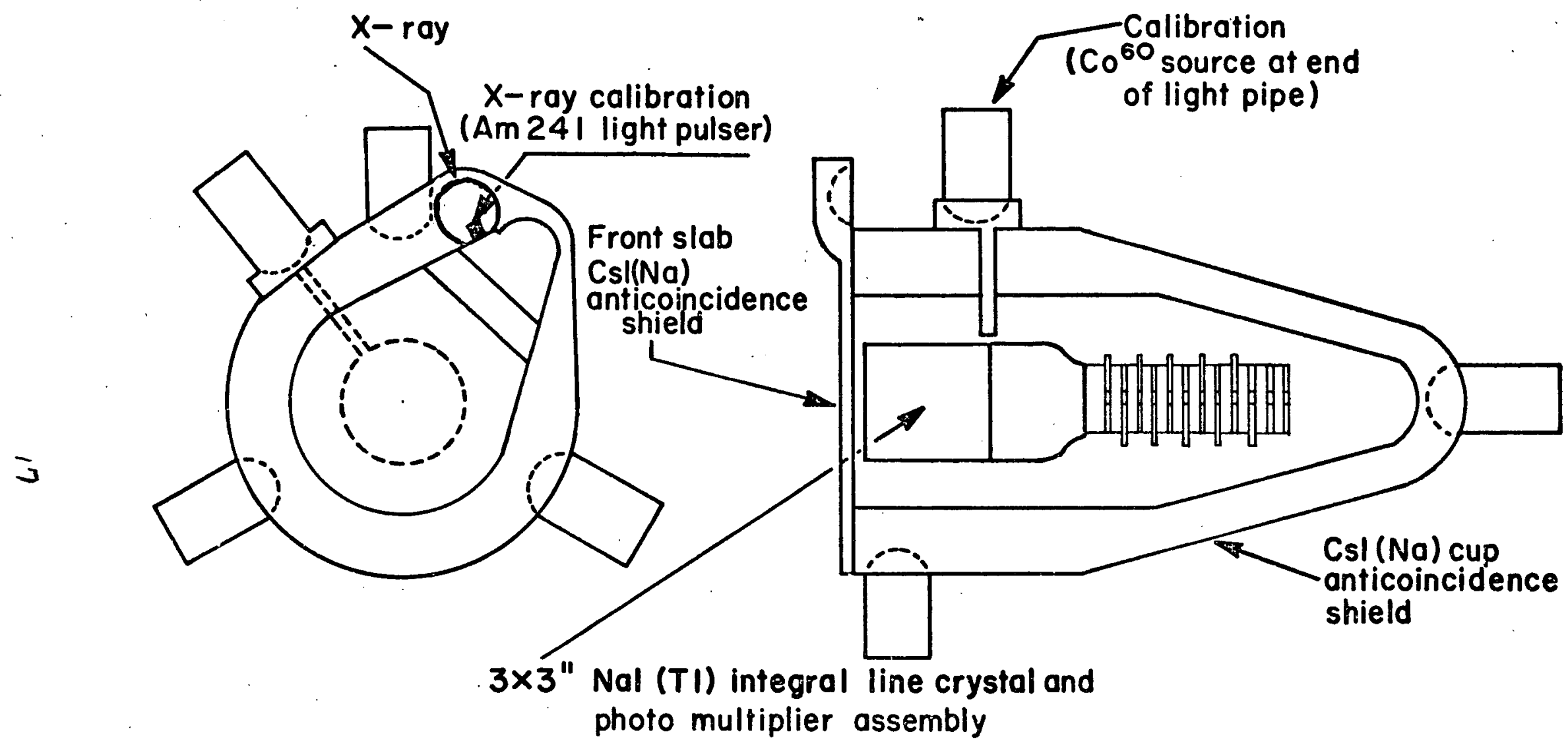

FIGURE ] 


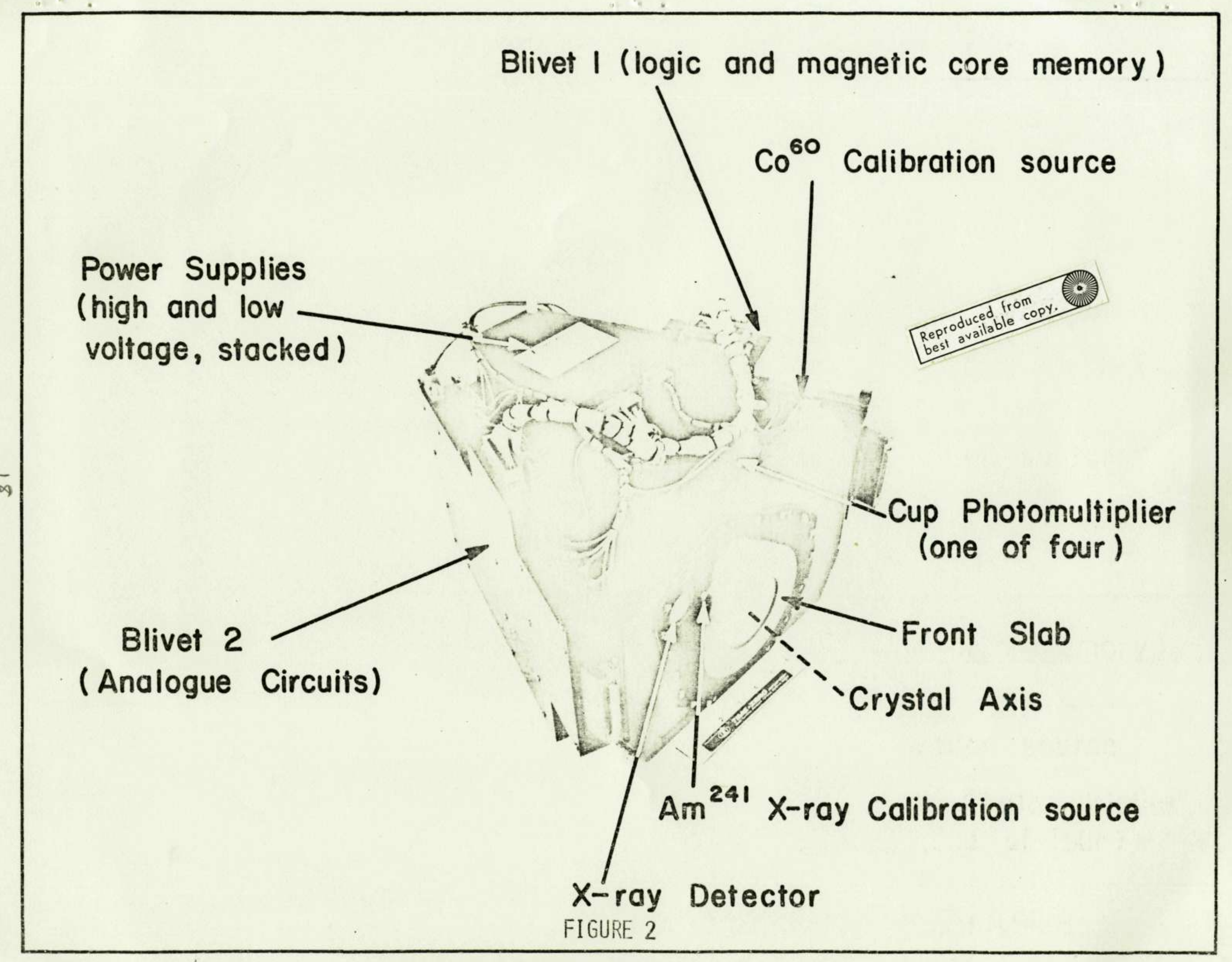




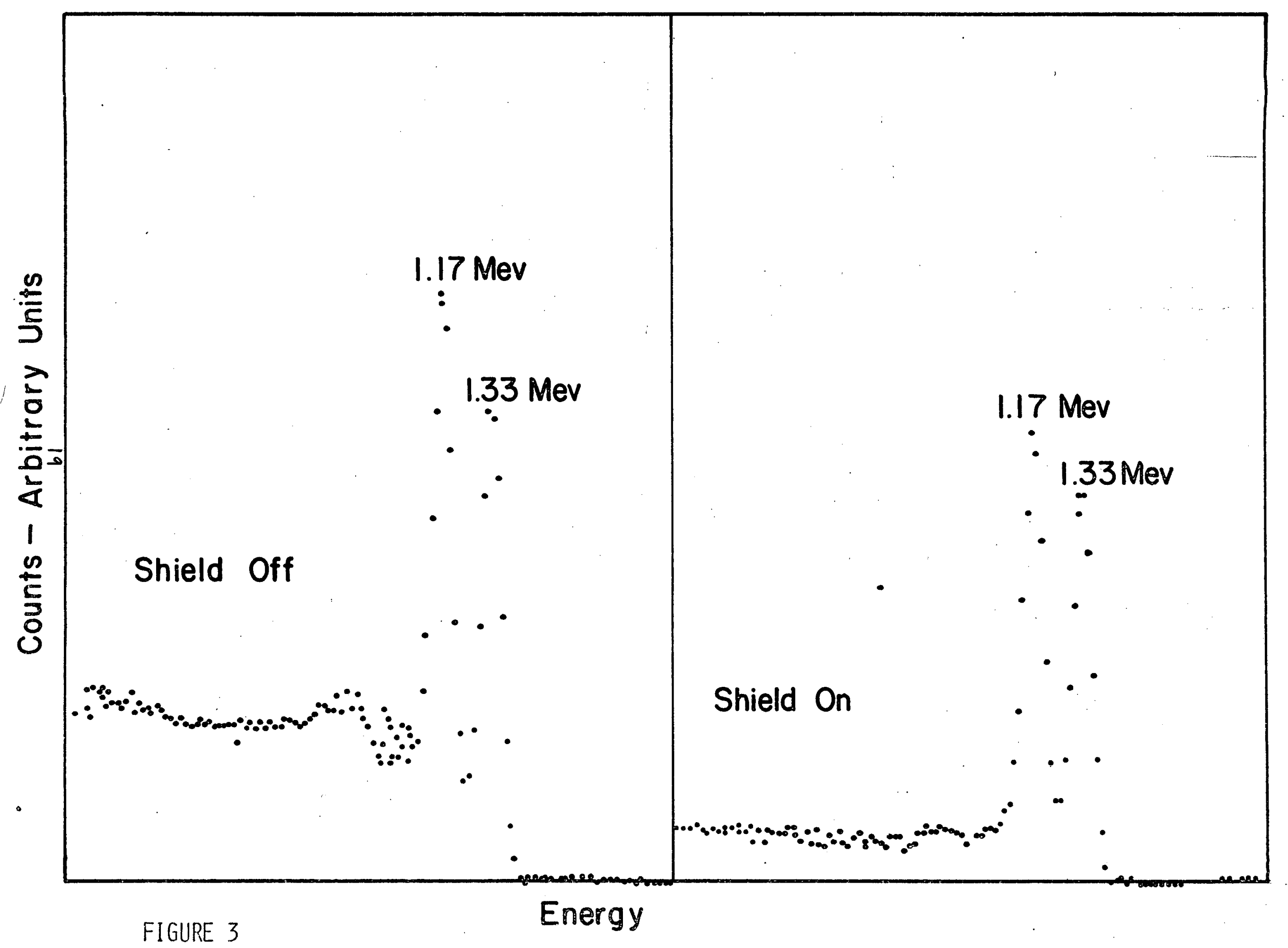




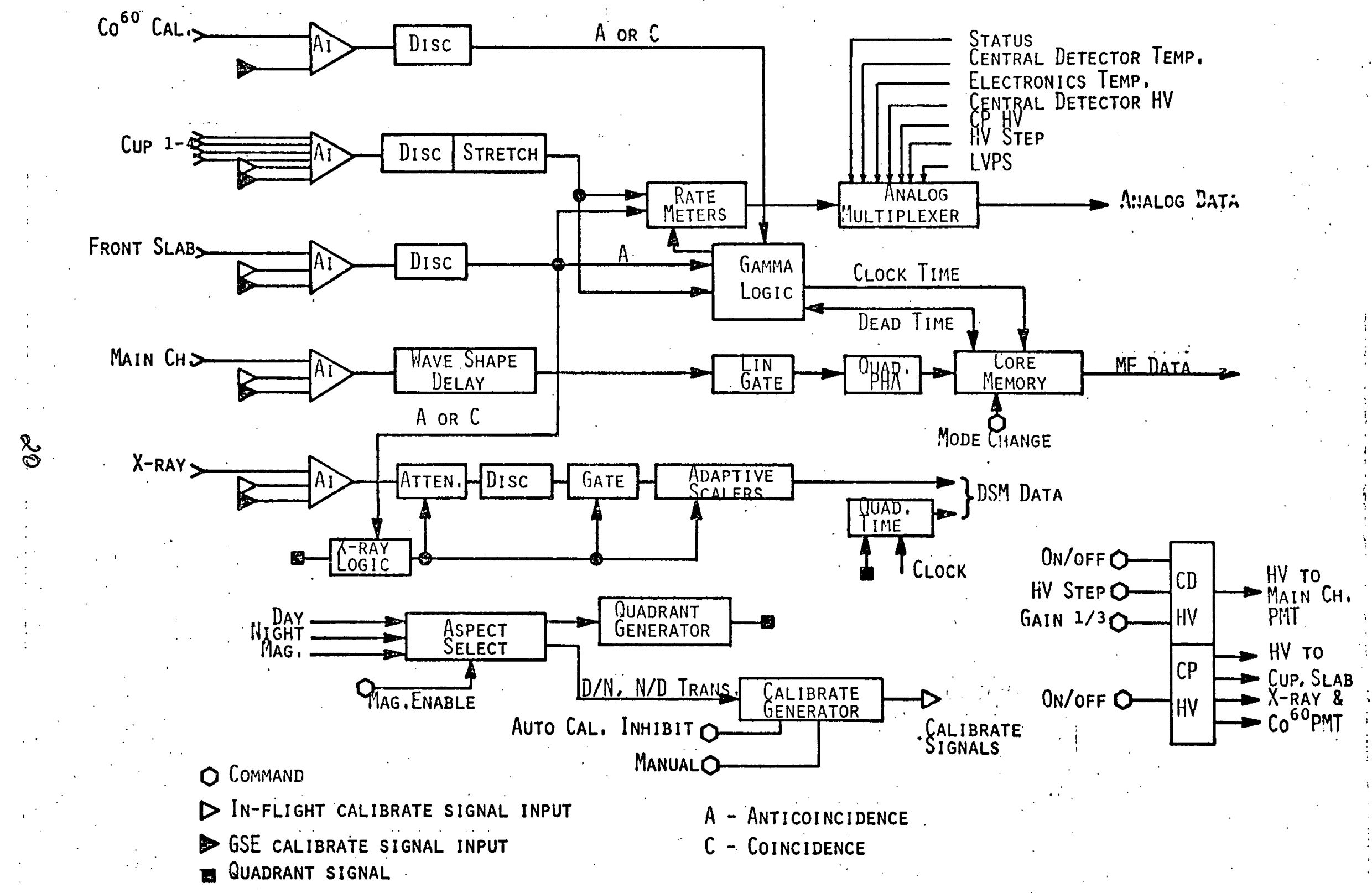

FIGIIRE 4 


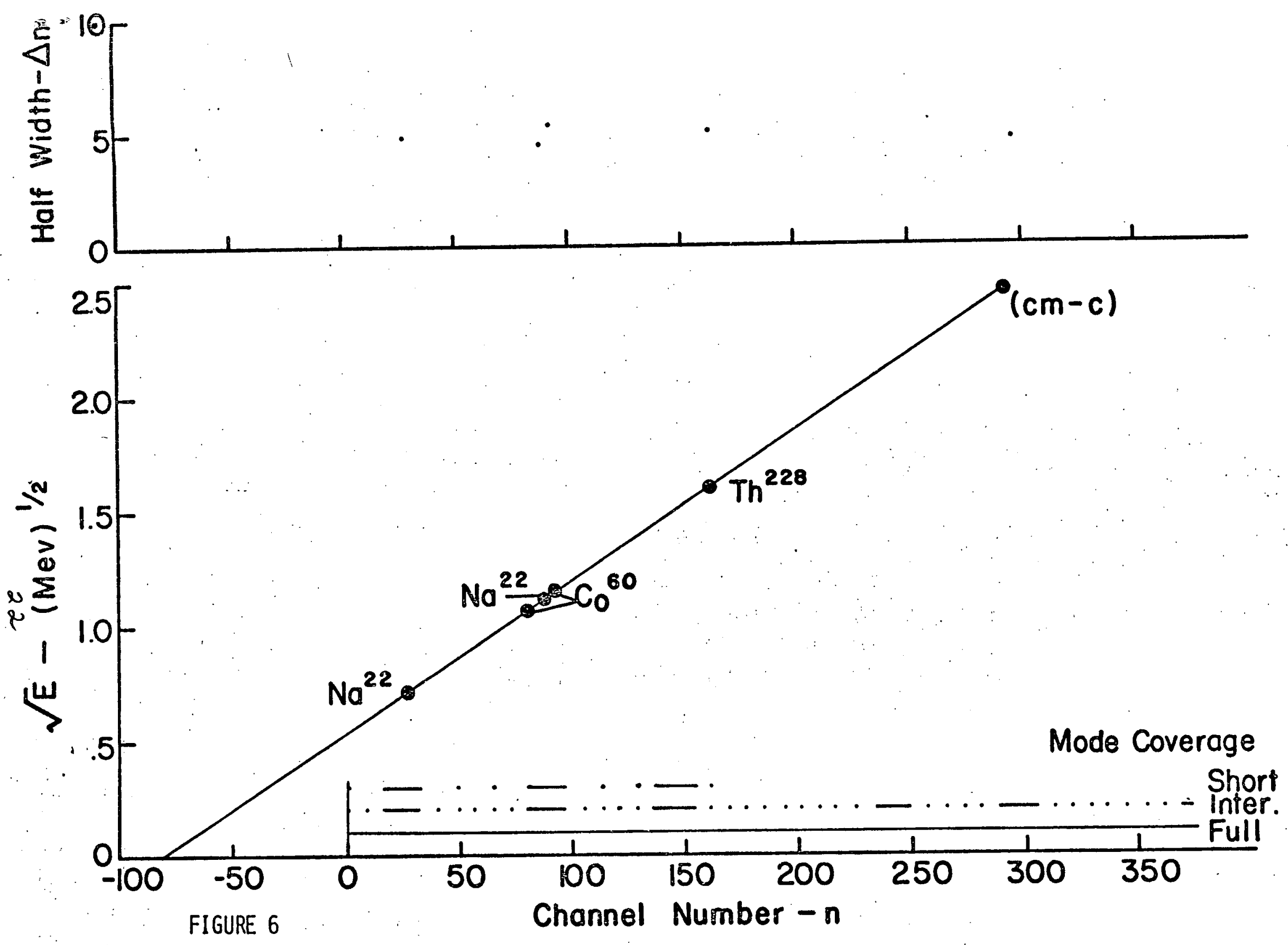

